\title{
Electric-field dependent spin diffusion and spin injection into semiconductors
}

\author{
Z. G. Yu and M. E. Flatté \\ Department of Physics and Astronomy, University of Iowa, Iowa City, Iowa 52242
}

(Dated: February 28, 2019)

\begin{abstract}
We derive a drift-diffusion equation for spin polarization in semiconductors by consistently taking into account electric-field effects and nondegenerate electron statistics. We identify a high-field diffusive regime which has no analogue in metals. In this regime there are two distinct spin diffusion lengths. Furthermore, spin injection from a ferromagnetic metal into a semiconductor is enhanced by several orders of magnitude and spins can be transported over distances much greater than the low-field spin diffusion length.

PACS numbers: 72.25.Dc, 72.20.Ht, 72.25.Hg, 72.25.Mk.
\end{abstract}

Semiconductor devices based on the control and manipulation of electron spin (semiconductor spintronics) have recently attracted considerable attention [1]. Spin transport and injection properties of semiconductors and heterostructures strongly constrain the design of new spintronic devices. In theoretical studies of spin transport and injection in semiconductors [2, 3, , 4] the spin polarization is usually assumed to obey the same diffusion equation as in metals [5],

$$
\nabla^{2}\left(\mu_{\uparrow}-\mu_{\downarrow}\right)-\left(\mu_{\uparrow}-\mu_{\downarrow}\right) / L^{2}=0,
$$

where $\mu_{\uparrow(\downarrow)}$ is the electrochemical potential of up-spin (down-spin) electrons. In this diffusion equation, the electric field does not play any role, and spin polarization decays away on a length scale of $L$ from an injection point. This is reasonable for metals because the electric field $\mathbf{E}$ is essentially screened. For semiconductor spintronic devices, however, the semiconductor often is lightly doped and nondegenerate, and moderate electric field can dominate the carrier motion. Equation (1) corresponds to neglecting drift in the more general driftdiffusion equation for the spin polarization,

$$
\nabla^{2}\left(n_{\uparrow}-n_{\downarrow}\right)+\frac{e \mathbf{E}}{k_{B} T} \cdot \nabla\left(n_{\uparrow}-n_{\downarrow}\right)-\frac{\left(n_{\uparrow}-n_{\downarrow}\right)}{\left(L^{(s)}\right)^{2}}=0,
$$

where $n_{\uparrow}-n_{\downarrow}$ is the difference between up-spin and downspin electron densities and $L^{(s)}$ is the intrinsic spin diffusion length.

If Eq. (1) holds, spin injection from a ferromagnetic metal to a semiconductor without a spin-selective interfacial barrier is virtually impossible due to the "conductivity mismatch", or more precisely, a mismatch between effective resistances in the metal $\left(L^{(f)} / \sigma_{f}\right)$ and in the semiconductor $\left(L^{(s)} / \sigma_{s}\right)$ [2, 3, 怔. Here $L^{(f)}$ and $L^{(s)}$ are the spin diffusion lengths for the ferromagnetic metal and the semiconductor, and $\sigma_{f}$ and $\sigma_{s}$ are conductivities for the two materials. Even for spin injection from ferromagnetic semiconductors, $L^{(f)} / \sigma_{f} \gg L^{(s)} / \sigma_{s}$, and the spin polarization is much less than $99 \%$, so the large spin injection percentages achieved from $\mathrm{ZnMnSe}$ [6, 7] and GaMnAs [8] are difficult to understand via Eq. (1).
Here we clarify the central role of the electric field on spin transport in semiconductors. We obtain the driftdiffusion equation (2) for the spin polarization in a semiconductor. Equation (2) consistently takes into account electric-field effects and nondegenerate electron statistics. We identify a high-field diffusive regime which has no analogue in metals. This regime occurs for field as small as $1 \mathrm{~V} / \mathrm{cm}$ at low temperatures. Two distinct spin diffusion lengths now characterize spin motion, i.e., upstream $\left(L_{u}\right)$ and down-stream $\left(L_{d}\right)$ spin diffusion lengths, which can differ in orders of magnitude with realistic fields: $E \geq 2.5 \mathrm{~V} / \mathrm{cm}$ at $T=3 \mathrm{~K}$ and $E \geq 250 \mathrm{~V} / \mathrm{cm}$ at $T=300 \mathrm{~K}$. These two length scales play distinctive but both favorable roles in spin injection from a ferromagnetic metal to a semiconductor. We find that the effective semiconductor resistance determining the injection efficiency is $L_{u} / \sigma_{s}$ rather than $L^{(s)} / \sigma_{s}$, which may be comparable to $L^{(f)} / \sigma_{f}$ given that $L_{u}$ can be shorter than $L^{(s)}$ by several orders of magnitude in the highfield regime. Moreover, the decay length scale for the spin polarization injected into the semiconductor is $L_{d}$, which would be much longer than $L^{(s)}$ in the presence of a strong field. Our results suggest a simple and practical approach to increase spin injection and spin coherence in semiconductors, namely, increasing the electric field, or equivalently, increasing the total injection current in semiconductors. Our results are consistent with the significant current dependence observed for spin injection from Fe to GaAs [9]. We further note that strong fields also substantially enhance spin injection in structures with an interfacial barrier.

The semiconductor we consider here is lightly or moderately $n$-doped ( $p$-doped semiconductors can be analyzed similarly), which is typical in spintronic devices. We assume that there is no space charge and the material is homogeneous. The current for up-spin and downspin can be written as $\mathbf{j}_{\uparrow(\downarrow)}=\sigma_{\uparrow(\downarrow)} \mathbf{E}+e D \nabla n_{\uparrow(\downarrow)}$, which consists of the drift current and the diffusion one. Here $D$ is the electron diffusion constant, $\sigma_{\uparrow(\downarrow)}$ the up-spin (down-spin) conductivity, and $n_{\uparrow(\downarrow)}$ the up-spin (downspin) electron density. The spin-dependent conductivity is proportional to the electron density for individual 


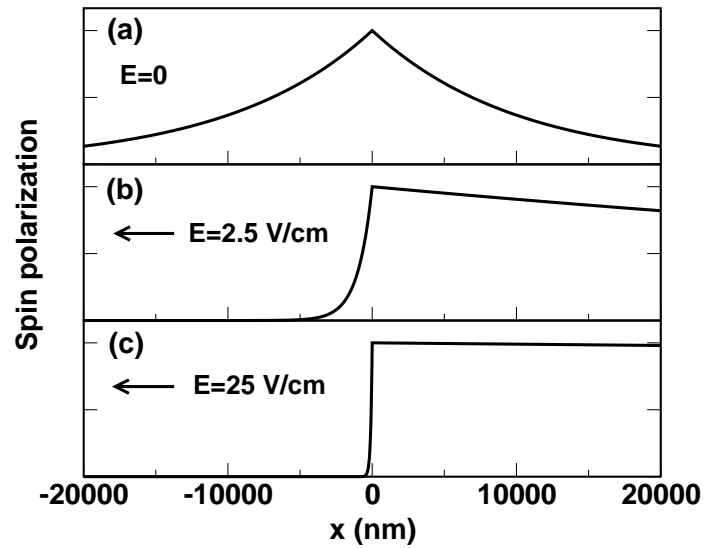

FIG. 1: Distribution of electron spin polarization as a function of position for a spin imbalance injected at $x=0$. Panel (a), (b), and (c) are for $|e E| / k_{B} T=0,0.001$, and $0.01 \mathrm{~nm}^{-1}$, which correspond to $E=0,2.5 \mathrm{~V} / \mathrm{cm}$, and $25 \mathrm{~V} / \mathrm{cm}$, respectively, at $T=3 \mathrm{~K}$. The intrinsic spin diffusion length is $L^{(s)}=10^{4} \mathrm{~nm}$.

spins, $\sigma_{\uparrow(\downarrow)}=n_{\uparrow(\downarrow)} e \nu_{e}$, where the mobility $\nu_{e}$ is assumed to be independent of field and density. The rate at which spin-up (spin-down) electrons scatter to spin-down (spinup) electrons is denoted by $1 / \tau_{\uparrow \downarrow}\left(1 / \tau_{\downarrow \uparrow}\right)$. In steady state, the equations of continuity for individual spins read

$\nabla \cdot \mathbf{j}_{\uparrow}=\nabla \sigma_{\uparrow} \cdot \mathbf{E}+\sigma_{\uparrow} \nabla \cdot \mathbf{E}+e D \nabla^{2} n_{\uparrow}=\left(\frac{n_{\uparrow}}{\tau_{\uparrow \downarrow}}-\frac{n_{\downarrow}}{\tau_{\downarrow \uparrow}}\right) e$,
$\nabla \cdot \mathbf{j}_{\downarrow}=\nabla \sigma_{\downarrow} \cdot \mathbf{E}+\sigma_{\downarrow} \nabla \cdot \mathbf{E}+e D \nabla^{2} n_{\downarrow}=\left(\frac{n_{\downarrow}}{\tau_{\downarrow \uparrow}}-\frac{n_{\uparrow}}{\tau_{\uparrow \downarrow}}\right) e$.

In nondegenerate semiconductors, $\tau_{\uparrow \downarrow}^{-1}=\tau_{\downarrow \uparrow}^{-1} \equiv \tau^{-1} / 2$.

For a homogeneous semiconductor without spacecharge, local variation of electron density $\Delta n$ should be balanced by a local change of hole concentration $\Delta p$. In doped semiconductors, spin polarization can be created without changing electrons or hole densities $\Delta n=\Delta p=$ 0 [10, and therefore,

$$
\Delta n_{\uparrow}+\Delta n_{\downarrow}=0 .
$$

Here $\Delta n_{\uparrow(\downarrow)}=n_{\uparrow(\downarrow)}-n_{0} / 2$, and $n_{0}$ is the total electron density in equilibrium. From Poisson's equation, $\nabla \cdot \mathbf{E}=-\left(\Delta n_{\uparrow}+\Delta n_{\downarrow}\right) e / \epsilon=0$. By using the Einstein's relation, $D=k_{B} T \nu_{e} / e$, where $k_{B}$ is the Boltzmann constant and $T$ is temperature, we obtain the differential equation (2) for $n_{\uparrow}-n_{\downarrow}$, the measure of the spin polarization in semiconductors, with $L^{(s)}=\sqrt{D \tau}$.

Equation (2), together with the local charge neutrality constraint Eq. (3), dramatically alters the spin transport behavior in semiconductors from that expected from Eq. (1). The general form of solution to Eq. (2) (restricting variation to the $x$-direction) is

$$
n_{\uparrow}-n_{\downarrow}=A \exp \left(-x / L_{1}\right)+B \exp \left(-x / L_{2}\right),
$$

where $\lambda_{1}=1 / L_{1}$ and $\lambda_{2}=1 / L_{2}$ are the roots of the quadratic equation, $\lambda^{2}-\lambda e E / k_{B} T-1 /\left(L^{(s)}\right)^{2}=0$. One of



FIG. 2: Up-stream (dashed line) and down-stream (solid line) diffusion lengths as a function of electric field. The intrinsic spin diffusion length is $L^{(s)}=10^{4} \mathrm{~nm}$.

the roots of the above equation must be positive and the other negative. The choice of roots is constrained by the boundary conditions at $\pm \infty$. To understand the physical consequence of the electric field on the spin transport, we suppose that a continuous spin imbalance is injected at $x=0$, and the electric field is along the $-x$ direction. The spin polarization will gradually decay in size as the distance from the point of injection increases. In Fig. 1, we plot the spin polarization as a function of position for different fields. In the absence of the field, as shown in Fig. 1(a), the spin polarization decays symmetrically along $-x$ and $+x$ with a single length scale, $L^{(s)}$. When an electric field is applied, the decay of the spin polarization becomes spatially asymmetric. For spin diffusion opposite to the field direction (down-stream for electrons), the decay length of the spin polarization is longer than $L^{(s)}$. For spin diffusion along the field direction (up-stream for electrons), the decay length is shorter than $L^{(s)}$. As we change the strength of the field, the spatial distribution of the spin polarization can change dramatically.

We define two quantities $L_{d}, L_{u}$,

$$
\begin{gathered}
L_{d}=\left[-\frac{|e E|}{2 k_{B} T}+\sqrt{\left(\frac{e E}{2 k_{B} T}\right)^{2}+\frac{1}{\left(L^{(s)}\right)^{2}}}\right]^{-1}, \\
L_{u}=\left[\frac{|e E|}{2 k_{B} T}+\sqrt{\left(\frac{e E}{2 k_{B} T}\right)^{2}+\frac{1}{\left(L^{(s)}\right)^{2}}}\right]^{-1} .
\end{gathered}
$$

The distribution of the spin polarization in Fig. 1 is then described by $n_{\uparrow}-n_{\downarrow} \sim \exp \left(-x / L_{d}\right)$ for $x>0$, and $n_{\uparrow}-n_{\downarrow} \sim \exp \left(x / L_{u}\right)$ for $x<0$. Thus $L_{d}\left(L_{d}>L^{(s)}\right)$ and $L_{u}\left(L_{u}<L^{(s)}\right)$ are the down-stream and up-stream spin diffusion lengths, respectively.

Figure 2 shows $L_{d}$ and $L_{u}$ as a function of the electric field. In the absence of the field, the down-stream and up-stream lengths are equal to the intrinsic diffusion length $L^{(s)}$. With increasing field the down-stream 
diffusion length $L_{d}$ increases, whereas the up-stream diffusion length $L_{u}$ decreases. A high-field regime for spin transport in semiconductors can be defined by $E>E_{c}$, where $e E_{c} / k_{B} T=1 / L^{(s)}$. In this regime, $L_{u}$ and $L_{d}$ deviate from $L^{(s)}$ considerably and the spin diffusion behavior is qualitatively different from that in low fields. We emphasize that since $L^{(s)}$ is large in semiconductors, this regime is not beyond realistic fields where most spintronic devices operate. For a typical spin diffusion length, $L^{(s)}=10^{4} \mathrm{~nm} \mathrm{[11],} E_{c}=25 \mathrm{~V} / \mathrm{cm}$ at $T=300 \mathrm{~K}$ and $E_{c}=0.25 \mathrm{~V} / \mathrm{cm}$ at $T=3 \mathrm{~K}$.

The physics of the field effects on the spin diffusion becomes clearer at the strong-field limit, where $|e E| / K_{B} T \gg 1 / L^{(s)}$. In this limit, the electrons move with velocity $|E| \nu_{e}$ and so does the spin polarization. $L_{d}$ is simply the distance over which the carriers move within the spin life time $\tau, L_{d} \simeq|E| \nu_{e} \tau=|E| \frac{e}{k_{B} T} D \tau=$ $\left(L^{(s)}\right)^{2}|e E| / k_{B} T$. For the up-stream diffusion length $L_{u}$ at this limit, $L_{u} \simeq k_{B} T /|e E|$, which simply corresponds to a Boltzmann distribution of electrons in a retarding field.

A similar field-dependent diffusion phenomenon has been observed and studied in charge transport of minority carriers in doped semiconductors 12]. In fact, if $n_{\uparrow}-n_{\downarrow}$ is substituted by $\Delta p$ and $L^{(s)}$ is regarded as the intrinsic charge diffusion length, Eq. (2) becomes the diffusion equation for the disturbance of minority carrier in $n$-doped semiconductors. It is known that the electric field leads to two distinct charge diffusion lengths in this case as well as a modification of carrier injection [12].

As an application of our field-dependent spin transport theory, we study how the electric field affects spin injection from a ferromagnetic metal to a semiconductor. We consider a simple one-dimensional spin injection structure to elucidate the underlying physics of electric field and nondegenerate electron statistics effects. This injection structure, as shown in the inset of Fig. 3, comprises a semi-infinite metal $(x<0)$ and a semi-infinite semiconductor $(x>0)$. Electrons are injected from the metal to the semiconductor, and therefore, the electric field is antiparallel to the $x$-axis. In the ferromagnetic metal the electrochemical potentials for individual spins satisfy the equations 13,

$$
\frac{d^{2}}{d x^{2}}\left(\begin{array}{c}
\mu_{\uparrow} \\
\mu_{\downarrow}
\end{array}\right)=\left(\begin{array}{cc}
\left(D_{\uparrow}^{f} \tau_{\uparrow \downarrow}\right)^{-1} & -\left(D_{\uparrow}^{f} \tau_{\uparrow \downarrow}\right)^{-1} \\
-\left(D_{\downarrow}^{f} \tau_{\downarrow \uparrow}\right)^{-1} & \left(D_{\downarrow}^{f} \tau_{\downarrow \uparrow}\right)^{-1}
\end{array}\right)\left(\begin{array}{c}
\mu_{\uparrow} \\
\mu_{\downarrow}
\end{array}\right),
$$

where $D_{\uparrow(\downarrow)}^{f}$ is the up-spin (down-spin) electron diffusion constant. In metals the conductivity and the diffusion constant are related via $\sigma_{\uparrow(\downarrow)}^{f} / D_{\uparrow(\downarrow)}^{f}=e^{2} N_{\uparrow(\downarrow)}\left(E_{F}\right)$, and $N_{\uparrow(\downarrow)}\left(E_{F}\right)$ is the up-spin (down-spin) density of states at Fermi energy. It is readily seen that the above equations lead to Eq. (1) if $L^{(f)}=\left[\left(D_{\uparrow}^{f} \tau_{\uparrow \downarrow}\right)^{-1}+\left(D_{\downarrow}^{f} \tau_{\downarrow \uparrow}\right)^{-1}\right]^{-1 / 2}$.

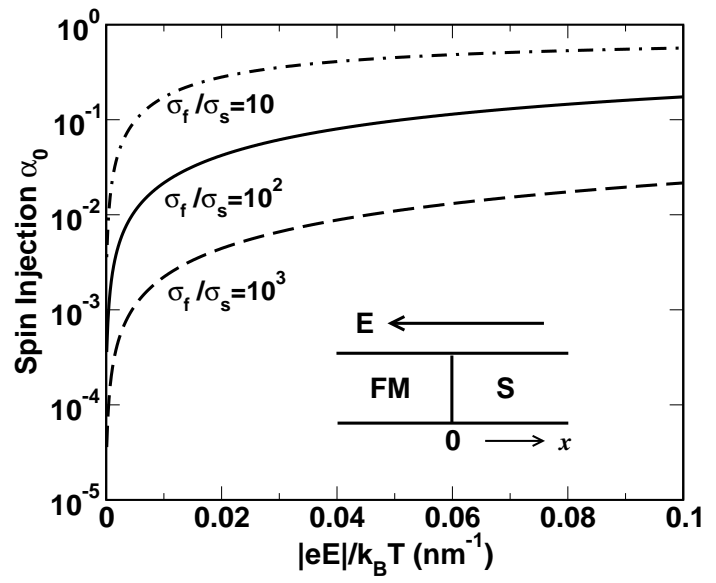

FIG. 3: Spin injection efficiency $\alpha_{0}$ as a function of electric field. Dot-dashed, solid, and dashed lines correspond to $\sigma_{f} / \sigma_{s}=10,100$, and 1000, respectively. Other parameters are $p_{f}=0.8, L^{(f)}=100 \mathrm{~nm}, L^{(s)}=10^{4} \mathrm{~nm}$. The inset shows the schematic injection structure.

The general solution can be written as

$$
\frac{1}{e J}\left(\begin{array}{c}
\mu_{\uparrow} \\
\mu_{\downarrow}
\end{array}\right)=\frac{x}{\sigma_{\uparrow}^{f}+\sigma_{\downarrow}^{f}}\left(\begin{array}{l}
1 \\
1
\end{array}\right)+C_{1} e^{\frac{x}{L^{(f)}}}\left(\begin{array}{c}
1 / \sigma_{\uparrow}^{f} \\
-1 / \sigma_{\downarrow}^{f}
\end{array}\right),
$$

where $J$ is the total electron current, which is a constant throughout the structure in steady state. In the semiconductor, according to Eqs. (2) and (3),

$$
\Delta n_{\uparrow}=-\Delta n_{\downarrow}=C_{2} \exp \left(-x / L_{d}\right),
$$

and $J=\sigma_{s} E$. In order to match boundary conditions at the interface between the metal and the semiconductor, it is desirable to know the electrochemical potentials for up-spin and down-spin electrons in the semiconductor, which are related to the electron density for individual spins via

$$
\mu_{\uparrow(\downarrow)}=k_{B} T \ln \left(1+\frac{2 \Delta n_{\uparrow(\downarrow)}}{n_{0}}\right)+e E x-C_{0} .
$$

This relation can be readily derived based on the definition of the electrochemical potential in nondegenerate semiconductors $n_{\uparrow(\downarrow)} \propto \exp \left[\left(\mu_{\uparrow(\downarrow)}+e \psi\right) / k_{B} T\right]$, where $E \equiv-d \psi / d x$.

The three unknown coefficients $C_{i}(i=0,1,2)$ in Eqs. (6)-(8) will be determined by the boundary conditions at the interface. For a clean and transparent interface, i.e., no spin-flip scattering at the interface and no interface resistance, both the electrochemical potential and the current for individual spins are continuous, giving rise to three independent equations: (1) $\mu_{\uparrow}\left(0^{-}\right)=\mu_{\uparrow}\left(0^{+}\right),(2) \mu_{\downarrow}\left(0^{-}\right)=\mu_{\downarrow}\left(0^{+}\right)$, and $(3)$ $j_{\uparrow}\left(0^{-}\right)-j_{\downarrow}\left(0^{-}\right)=j_{\uparrow}\left(0^{+}\right)-j_{\downarrow}\left(0^{+}\right)$. The current can be calculated using $j_{\uparrow(\downarrow)}=\sigma_{\uparrow(\downarrow)} \frac{d\left(\mu_{\uparrow(\downarrow)} / e\right)}{d x}$.

The spin injection in the semiconductor is usually defined via the spin polarization of the current, $\alpha(x)=$ 
$\left[j_{\uparrow}(x)-j_{\downarrow}(x)\right] / J$, which is found to be proportional to the spin polarization of the electron density $n_{\uparrow}-n_{\downarrow}$,

$$
\alpha(x)=\frac{n_{\uparrow}(x)-n_{\downarrow}(x)}{n_{0}}\left(1-\frac{k_{B} T}{e E L_{d}}\right) .
$$

Thus the solution of $n_{\uparrow}-n_{\downarrow}$ in Eq. (7) indicates $\alpha(x)=$ $\alpha_{0} e^{-x / L_{d}}$, where $\alpha_{0}$ is the spin injection efficiency. We obtain an equation for $\alpha_{0}$, noting $1-k_{B} T / e E L_{d}=$ $-k_{B} T / e E L_{u}$,

$$
\frac{2 L^{(f)}\left(\alpha_{0}-p_{f}\right)}{\left(1-p_{f}^{2}\right) \sigma_{f}}=\frac{k_{B} T}{e E \sigma_{s}} \ln \frac{-k_{B} T / e E L_{u}+\alpha_{0}}{-k_{B} T / e E L_{u}-\alpha_{0}}
$$

where $\sigma_{f}=\sigma_{\uparrow}^{f}+\sigma_{\downarrow}^{f}$, and $p_{f}=\left(\sigma_{\uparrow}^{f}-\sigma_{\downarrow}^{f}\right) / \sigma_{f}$ is the spin polarization in the metal. We solve Eq. (10) and plot the spin injection efficiency $\alpha_{0}$ as a function of the electric field in Fig. 3. We see that the electric field can enhance the spin injection efficiency considerably. When $\Delta n_{\uparrow(\downarrow)} / n_{0} \ll 1$, i.e., small spin polarization in the semiconductor, $\alpha(x)$ can be expressed in a compact form,

$$
\alpha(x)=\left[\frac{L^{(f)}}{\left(1-p_{f}^{2}\right) \sigma_{f}}+\frac{L_{u}}{\sigma_{s}}\right]^{-1} \frac{p_{f} L^{(f)}}{\left(1-p_{f}^{2}\right) \sigma_{f}} e^{-\frac{x}{L_{d}}} .
$$

This remarkable expression shows that the electricfield effects on spin injection can be described in terms of the two field-induced diffusion lengths. Both diffusion lengths affect spin injection favorably but in a different manner. The up-stream length $L_{u}$ controls the relevant resistance in the semiconductor, which determines the spin injection efficiency. With increasing field this effective resistance, $L_{u} / \sigma_{s}$, becomes smaller, and accordingly the spin injection efficiency is enhanced. The transport distance of the injected spin polarization in the semiconductor, however, is controlled by the down-stream length $L_{d}$. As the field increases, this distance becomes longer.

We now contrast Eq. (11) with that obtained by previous calculations 2, 3, 4] based on Eq. (1). The spin injection

$$
\alpha(x)=\left[\frac{L^{(f)}}{\left(1-p_{f}^{2}\right) \sigma_{f}}+\frac{L^{(s)}}{\sigma_{s}}\right]^{-1} \frac{p_{f} L^{(f)}}{\left(1-p_{f}^{2}\right) \sigma_{f}} e^{-\frac{x}{L^{(s)}}}
$$

is given by the zero-field result of Eq. (11). As $L^{(f)} \ll$ $L^{(s)}$ and $\sigma_{f} \gg \sigma_{s}$, the effective resistance in the metal, $L^{(f)} / \sigma_{f}$, is much less than its counterpart in the semiconductor, $L^{(s)} / \sigma_{s}$. Thus Eq. (12) suggests that this resistance mismatch makes it virtually impossible to realize an appreciable spin injection from a ferromagnetic metal to a semiconductor. However, the more general description of the spin transport in semiconductors indicates that the effective semiconductor resistance to be compared with $L^{(f)} / \sigma_{f}$ should be $L_{u} / \sigma_{s}$ rather than $L^{(s)} / \sigma_{s}$. Since $L_{u}$ can be smaller than $L^{(s)}$ by orders of magnitude in the high-field regime, this "conductivity mismatch" obstacle may be overcome with the help of strong electric fields, or equivalently, large injection currents [14]. For example, if the parameters of a spin injection device are as follows, $p_{f}=0.8, L^{(f)}=100 \mathrm{~nm}$, $L^{(s)}=10^{4} \mathrm{~nm}$, and $\sigma_{f}=100 \sigma_{s}$, at zero field the spin injection efficiency is $0.04 \%$, which can be increased to $4.2 \%$ at $|e E| / k_{B} T=0.02 \mathrm{~nm}^{-1}$, which corresponds to $|E|=50 \mathrm{~V} / \mathrm{cm}$, or $|J|=50 \mathrm{~A} / \mathrm{cm}^{2}$ for a typical semiconductor conductivity $\sigma_{s}=1(\Omega \mathrm{cm})^{-1}$, at $T=3 \mathrm{~K}$. This may explain the large spin injection percentages from ZnMnSe to ZnSe [6, 7] and from Fe to GaAs [9, 15], as well as the dramatic increase in spin injection with current in Ref. [9]. Finally, we note that spin injection enhancement from a spin-selective interfacial barrier between the ferromagnetic metal and the semiconductor,

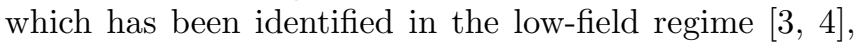
becomes more pronounced in the high-field regime.

In summary, we have derived the drift-diffusion equation for spin polarization in a semiconductor by consistently taking into account electric-field effects and nondegenerate electron statistics. This equation provides a framework to understand spin transport in semiconductors. We have identified a high-field diffusive regime which has no analogue in metals. In this regime, there are two distinct spin diffusion lengths, i.e., the up-stream and down-stream spin diffusion lengths. The high-field description of the spin transport in semiconductors predicts that the electric field can effectively enhance spin injection from a ferromagnetic metal into a semiconductor and substantially increase the transport distance of the spin polarization in semiconductors. Our results suggest that the "conductivity mismatch" obstacle in spin injection may be overcome with the help of high field injection in the diffusive regime.

We would like to thank N. Samarth for pointing out the relevance of resistance mismatch for spin injection from ferromagnetic semiconductors. This work was supported by DARPA/ARO DAAD19-01-0490.
[1] See, e.g., S. A. Wolf et al., Science, 294, 1488 (2001), and references therein.

[2] G. Schmidt et al., Phys. Rev. B 62, R4790 (2000).

[3] E. I. Rashba, Phys. Rev. B 62, R16267 (2000).

[4] D. L. Smith and R. N. Silver, Phys. Rev. B 64, 045323 (2001).
[5] P. C. van Son, H. van Kempen, and P. Wyder, Phys. Rev. Lett. 58, 2271 (1987).

[6] R. Flederling et al., Nature (London) 402, 787 (1999).

[7] B. T. Jonker et al., Phys. Rev. B 62, 8180 (2000).

[8] Y. Ohno et al., Nature (London) 402, 790 (1999).

[9] A. T. Hanbicki et al., cond-mat/0110059. 
[10] J. M. Kikkawa and D. D. Awschalom, Phys. Rev. Lett. 80, 4313 (1998); J. M. Kikkawa, I. P. Smorchkova, N. Samarth, and D. D. Awschalom, Science 277, 1284 (1997); M. E. Flatté and J. M. Byers, Phys. Rev. Lett. 84, 4220 (2000).

[11] J. M. Kikkawa and D. D. Awschalom, Nature (London) 397, 139 (1999).

[12] See, e.g., R. A. Smith, Semiconductors (University Press, Cambridge, England, 1959).
[13] S. Hershfield and H. L. Zhao, Phys. Rev. B 56, 3296 (1997).

[14] Although it has been realized that spin injection can be enhanced by increasing the total injection current [4], the electric-field effects on spin transport were not taken into account and therefore the physics of the high-field regime was not captured.

[15] H. J. Zhu et al., Phys. Rev. Lett. 87, 016601 (2001). 